\title{
Declined Preoperative Aspartate Aminotransferase to Neutrophil Ratio Index Predicts Poor Prognosis in Patients with Intrahepatic Cholangiocarcinoma after Hepatectomy
}

\author{
Lingyun Liu, MD ${ }^{1}$ \\ Wei Wang, MD2 \\ Yi Zhang, MD 1 \\ Jianting Long, $\mathrm{MD}^{3}$ \\ Zhaohui Zhang, MD' \\ Qiao Li, MD' \\ Bin Chen, MD \\ Shaoqiang Li, MD' \\ Yunpeng Hua, $\mathrm{MD}^{1}$ \\ Shunli Shen, MD, PhD' \\ Baogang Peng, MD, PhD
}

\author{
${ }^{1}$ Department of Hepatic Surgery, \\ The First Affiliated Hospital of \\ Sun Yat-sen University, Guangzhou, \\ ${ }^{2}$ Department of Medical Ultrasonics, Institute \\ of Diagnostic and Interventional Ultrasound, \\ The First Affiliated Hospital of \\ Sun Yat-sen University, Guangzhou, \\ ${ }^{3}$ Department of Oncology, \\ The First Affiliated Hospital of \\ Sun Yat-sen University, Guangzhou, China
}

Correspondence: Baogang Peng, MD, PhD

Department of Hepatic Surgery,

The First Affiliated Hospital of Sun Yat-sen

University, No. 58, Zhong Shan Road 2,

Guangzhou 510080, Guangdong, China

Tel: 86-20-87755766-8214

Fax: 86-20-87755766-8663

E-mail: pengbaogang@medmail.com.cn

Co-correspondence: Shunli Shen, MD, PhD Department of Hepatic Surgery,

The First Affiliated Hospital of Sun Yat-sen University, No. 58, Zhong Shan Road 2, Guangzhou 510080, Guangdong, China

Tel: 86-20-87755766-8214

Fax: 86-20-87755766-8663

E-mail: shunlishen@163.com

Received March 2, 2017

Accepted May 24, 2017

Published Online June 1, 2017

${ }^{*}$ Lingyun Liu and Wei Wang contributed equally to this work.

\section{Purpose}

Various inflammation-based prognostic biomarkers such as the platelet to lymphocyte ratio and neutrophil to lymphocyte ratio, are related to poor survival in patients with intrahepatic cholangiocarcinoma (ICC). This study aims to investigate the prognostic value of the aspartate aminotransferase to neutrophil ratio index (ANRI) in ICC after hepatic resection.

\section{Materials and Methods}

Data of 184 patients with ICC after hepatectomy were retrospectively reviewed. The cut-off value of ANRI was determined by a receiver operating characteristic curve. Preoperative ANRI and clinicopathological variables were analyzed. The predictive value of preoperative ANRI for prognosis of ICC was identified by univariate and multivariate analyses.

\section{Results}

The optimal cut-off value of ANRI was 6.7. ANRI was associated with tumor size, tumor recurrence, white blood cell, neutrophil count, aspartate aminotransferase, and alanine transaminase. Univariate analysis showed that ANRI, sex, tumor number, tumor size, tumor differentiation, lymph node metastasis, resection margin, clinical TNM stage, neutrophil count, and carcinoembryonic antigen were markedly correlated with overall survival (OS) and disease-free survival (DFS) in patients with ICC. Multivariable analyses revealed that ANRI, a tumor size $>6 \mathrm{~cm}$, poor tumor differentiation, and an R1 resection margin were independent prognostic factors for both OS and DFS. Additionally, preoperative ANRI also had a significant value to predict prognosis in various subgroups of ICC, including serum hepatitis B surface antigen-negative and preoperative elevated carbohydrate antigen 19-9 patients.

\section{Conclusion}

Preoperative declined ANRI is a noninvasive, simple, and effective predictor of poor prognosis in patients with ICC after hepatectomy.

\section{Key words}

Aspartate aminotransferase, Neutrophil,

Cholangiocarcinoma, Prognosis, Biomarkers 


\section{Introduction}

Intrahepatic cholangiocarcinoma (ICC) is the second most common biliary malignancy and accounts for $5 \%$ to $10 \%$ of primary liver carcinomas [1]. A rapidly increasing incidence of ICC has been reported over the last few decades [2]. Operation is the only potentially curative therapeutic measure for patients with ICC. Unfortunately, less than $30 \%$ of ICC patients are not able to have an operation at the initial diagnosis due to a lack of appropriate markers for early diagnosis. In addition, even after curative hepatectomy, the outcome remains grim, with the 5-year survival rate ranging from $20 \%$ to $40 \%$ [3,4]. The poor prognosis after surgery is mainly due to tumor recurrence and metastasis [5]. Therefore, it is of great significance to screen for effective markers to identify ICC patients at a high risk of recurrence or metastases, thus providing timely and effective therapeutic treatment to improve the clinical outcomes.

As a routine liver function test index, aspartate aminotransferase (AST) reflects the damage of the liver. It is commonly used to assess the severity of various liver diseases [6]. Recently, studies have reported that prognostic indexes based on inflammatory cells such as neutrophils and lymphocytes can reflect the survival of various malignancies $[7,8]$, with aspartate aminotransferase to neutrophil ratio index (ANRI) as one of them. For example, elevated ANRI was found to be significantly correlated with a poorer outcome in patients with hepatocellular carcinoma [9].

The relation between ANRI and the prognosis of ICC patients after hepatic resection has not been reported. In the present study, we aim to evaluate the predictive value of preoperative ANRI on both the overall survival (OS) and disease-free survival (DFS) of ICC patients after hepatectomy.

\section{Materials and Methods}

\section{Patients}

A total of 184 newly diagnosed ICC patients treated with hepatectomy at the First Affiliated Hospital, Sun Yat-sen University between April 2004 and September 2015 were included. All specimens were histologically proven to be ICC after surgery. Informed consent was obtained, and procedures were carried out with prior approval of the Ethics Committee of the First Affiliated Hospital of Sun Yat-sen University (Guangzhou, China). Routine assessment was performed for all patients within 7 days before surgery, including physical examination, complete hematologic and biochemistry profiles, chest X-ray, abdominal ultrasonography, and contrast-enhanced computed tomography (CT) scans or magnetic resonance imaging.

All patients were $>18$ years of age, with complete clinical and laboratory data. No patients had any coexistent hematological disorders or known active infection before treatment. In addition, patients with preoperative anti-tumor treatment, such as chemotherapy or percutaneous ablation, were excluded from this study.

\section{Treatment and follow-up}

Surgical strategies were decided by a multidisciplinary team meeting. Combined operations such as biliary-intestinal anastomosis were applied when needed. Postoperative follow-up of all 184 patients was regularly performed according to institutional practice, including serum carbohydrate antigen 19-9 (CA19-9), $\alpha$-fetoprotein (AFP), carcinoembryonic antigen (CEA), abdominal ultrasound, and chest $\mathrm{X}$-ray every 3 months, and contrast-enhanced CT every 6 months. Recurrence was defined as the emergence of clinical, radiological, and/or pathological diagnosis (tissues obtained by ultrasound-guided fine-needle aspiration) of the tumor. Patients with confirmed recurrence of ICC received salvage treatments, such as repeated hepatectomy, percutaneous ablation or chemotherapy. DFS was calculated from the date of operation to the date of recurrence. OS was calculated from the date of operation to the date of death or last followup.

\section{Statistical analysis}

The best cut-off value of ANRI was determined by a receiver operating characteristic (ROC) curve. The difference between categorical variables was test by the Pearson chisquare test (two-tailed). Pearson bivariate correlate analyses (two-tailed) were used to compare the correlations of these categorical variables. Non-normally distributed numerical variables were tested using a Mann-Whitney U test. Survival curves (including stratified analysis) were plotted using the Kaplan-Meier method and compared using the log-rank test. The Cox proportional hazards model (Backward stepwise) was used to determine the independent prognostic factors based on variables selected on univariate analysis (with $\mathrm{p}<0.05$ ), and the results were expressed as a hazard ratio with a $95 \%$ confidence interval $(95 \% \mathrm{CI})$. There are significant differences when $p$-values are $<0.05$, using two-tailed tests. Statistical analyses performed using SPSS ver. 20.0 (IBM Corp., Armonk, NY). 
Table 1. Relationship between ANRI and clinicopathological characteristics in ICC ( $\mathrm{n}=184)$

\begin{tabular}{|c|c|c|c|c|c|c|}
\hline \multirow{2}{*}{ Category } & \multirow{2}{*}{ No. } & \multicolumn{2}{|c|}{ ANRI } & \multirow{2}{*}{$\chi^{2}$} & \multirow{2}{*}{$\mathbf{r}$} & \multirow{2}{*}{ p-value } \\
\hline & & $\leq 6.7(\mathrm{n}=94)$ & $>6.7(n=90)$ & & & \\
\hline \multicolumn{7}{|l|}{ Age (yr) } \\
\hline$\leq 60$ & 117 & $59(50.4)$ & $58(49.6)$ & 0.056 & - & 0.813 \\
\hline$>60$ & 67 & $35(52.2)$ & $32(47.8)$ & & & \\
\hline \multicolumn{7}{|l|}{ Sex } \\
\hline Female & 85 & $40(47.1)$ & $45(52.9)$ & 1.026 & - & 0.311 \\
\hline Male & 99 & $54(54.5)$ & $45(45.5)$ & & & \\
\hline \multicolumn{7}{|c|}{ Preoperative symptom } \\
\hline No & 51 & $26(51.0)$ & $25(49.0)$ & 0.000 & - & 0.986 \\
\hline Yes & 133 & $68(51.1)$ & $65(48.9)$ & & & \\
\hline \multicolumn{7}{|l|}{ Cirrhosis } \\
\hline No & 138 & $70(50.7)$ & $68(49.3)$ & 0.029 & - & 0.865 \\
\hline Yes & 46 & $24(52.2)$ & $22(47.8)$ & & & \\
\hline \multicolumn{7}{|l|}{ HBsAg } \\
\hline Negative & 133 & $67(50.4)$ & $66(49.6)$ & 0.097 & - & 0.755 \\
\hline Positive & 51 & $27(52.9)$ & $24(47.1)$ & & & \\
\hline \multicolumn{7}{|c|}{ Tumor number } \\
\hline Single & 121 & $60(49.6)$ & $61(50.4)$ & 0.318 & - & 0.573 \\
\hline Multiple & 63 & $34(54.0)$ & $29(46.0)$ & & & \\
\hline \multicolumn{7}{|c|}{ Tumor size $(\mathrm{cm})^{\mathrm{a})}$} \\
\hline$\leq 6$ & 97 & $42(43.3)$ & $55(56.7)$ & 4.980 & -0.165 & 0.026 \\
\hline$>6$ & 87 & $52(59.8)$ & $35(40.2)$ & & & \\
\hline \multicolumn{7}{|l|}{ Capsulation } \\
\hline No & 111 & $58(52.3)$ & $53(47.7)$ & 0.152 & - & 0.697 \\
\hline Yes & 73 & $36(49.3)$ & $37(50.7)$ & & & \\
\hline \multicolumn{7}{|c|}{ Differentiation $^{\text {b) }}$} \\
\hline $\mathrm{W}+\mathrm{M}$ & 125 & $60(48.0)$ & $65(52.0)$ & 1.487 & - & 0.223 \\
\hline $\mathrm{P}$ & 59 & $34(57.6)$ & $25(42.4)$ & & & \\
\hline \multicolumn{7}{|c|}{ Lymph node metastasis } \\
\hline No & 111 & $51(45.9)$ & $60(54.1)$ & 2.959 & - & 0.085 \\
\hline Yes & 73 & $43(58.9)$ & $30(41.1)$ & & & \\
\hline \multicolumn{7}{|c|}{ Vascular invasion } \\
\hline No & 174 & $90(52.0)$ & $83(48.0)$ & 1.015 & - & 0.314 \\
\hline Yes & 11 & $4(36.4)$ & $7(63.6)$ & & & \\
\hline \multicolumn{7}{|l|}{ TNM $^{\mathrm{c}}$} \\
\hline $\mathrm{I}+\mathrm{II}$ & 79 & $39(49.4)$ & $40(50.6)$ & 0.164 & - & 0.686 \\
\hline III+IV & 105 & $55(52.4)$ & $50(47.6)$ & & & \\
\hline \multicolumn{7}{|c|}{ Resection margin } \\
\hline R0 & 108 & $60(55.6)$ & $48(44.4)$ & 2.089 & - & 0.148 \\
\hline $\mathrm{R} 1$ & 76 & $34(44.7)$ & $42(55.3)$ & & & \\
\hline \multicolumn{7}{|c|}{ Biliary-intestinal anastomosis } \\
\hline No & 158 & $83(52.5)$ & $75(47.5)$ & 0.934 & - & 0.334 \\
\hline Yes & 26 & $11(42.3)$ & $15(57.7)$ & & & \\
\hline \multicolumn{7}{|c|}{ Complication } \\
\hline No & 155 & $79(51.0)$ & $76(49.0)$ & 0.006 & - & 0.940 \\
\hline Yes & 29 & $15(51.7)$ & $14(48.3)$ & & & \\
\hline Intraoperati & & & & & & \\
\hline$\leq 400$ & 104 & $55(52.9)$ & $49(47.1)$ & 0.309 & - & 0.578 \\
\hline$>400$ & 80 & $39(48.8)$ & $41(51.2)$ & & & \\
\hline
\end{tabular}

(Continued to the next page) 
Table 1. Continued

\begin{tabular}{|c|c|c|c|c|c|c|}
\hline \multirow{2}{*}{ Category } & \multirow{2}{*}{ No. } & \multicolumn{2}{|c|}{ ANRI } & \multirow{2}{*}{$\chi^{2}$} & \multirow{2}{*}{$\mathbf{r}$} & \multirow{2}{*}{ p-value } \\
\hline & & $\leq 6.7(\mathrm{n}=94)$ & $>6.7(n=90)$ & & & \\
\hline \multicolumn{7}{|c|}{ Recurrence } \\
\hline No & 21 & $3(14.3)$ & $18(85.7)$ & 12.848 & -0.264 & $<0.001$ \\
\hline Yes & 163 & $91(55.8)$ & $72(44.2)$ & & & \\
\hline \multicolumn{7}{|c|}{ WBC $\left(\times 10^{9} / \mathrm{L}\right)$} \\
\hline$\leq 10$ & 148 & $63(42.6)$ & $85(57.4)$ & 21.971 & -0.346 & $<0.001$ \\
\hline$>10$ & 36 & $31(86.1)$ & 5 (13.9) & & & \\
\hline \multicolumn{7}{|c|}{ Neutrophil $\left(\times 10^{9} / \mathrm{L}\right)^{\mathrm{a})}$} \\
\hline$\leq 4.55$ & 93 & $30(32.3)$ & $63(67.7)$ & 26.679 & -0.381 & $<0.001$ \\
\hline$>4.55$ & 91 & $64(70.3)$ & $27(29.7)$ & & & \\
\hline \multicolumn{7}{|c|}{ Platelet $\left(\times 10^{9} / \mathrm{L}\right)$} \\
\hline$\leq 300$ & 139 & $66(47.5)$ & $73(52.5)$ & 2.956 & - & 0.086 \\
\hline$>300$ & 45 & $28(62.2)$ & $17(37.8)$ & & & \\
\hline \multicolumn{7}{|l|}{ AST (U/L) } \\
\hline$\leq 37$ & 133 & $89(66.9)$ & $44(33.1)$ & 48.122 & 0.511 & $<0.001$ \\
\hline$>37$ & 51 & $5(9.8)$ & $46(90.2)$ & & & \\
\hline \multicolumn{7}{|l|}{$\operatorname{ALT}(\mathrm{U} / \mathrm{L})$} \\
\hline$\leq 80$ & 158 & $91(57.6)$ & $67(42.4)$ & 18.952 & 0.321 & $<0.001$ \\
\hline$>80$ & 26 & 3 (11.5) & $23(88.5)$ & & & \\
\hline \multicolumn{7}{|c|}{$\gamma$-GT (U/L) } \\
\hline$\leq 50$ & 50 & $24(48.0)$ & $26(52.0)$ & 0.262 & - & 0.609 \\
\hline$>50$ & 134 & $70(52.2)$ & $64(47.8)$ & & & \\
\hline \multicolumn{7}{|l|}{$\operatorname{AFP}(\mu \mathrm{g} / \mathrm{L})$} \\
\hline$\leq 200$ & 176 & $89(50.6)$ & 87 (49.4) & 0.436 & - & 0.509 \\
\hline$>200$ & 8 & $5(62.5)$ & 3 (37.5) & & & \\
\hline \multicolumn{7}{|c|}{ CEA $(\mu \mathrm{g} / \mathrm{L})$} \\
\hline$\leq 5.0$ & 107 & $53(49.5)$ & $54(50.5)$ & 0.247 & - & 0.619 \\
\hline$>5.0$ & 77 & $41(53.2)$ & $36(46.8)$ & & & \\
\hline \multicolumn{7}{|c|}{ CA19-9 (U/mL) } \\
\hline$\leq 35$ & 65 & $37(56.9)$ & $28(43.1)$ & 1.370 & - & 0.242 \\
\hline$>35$ & 119 & 57 (47.9) & $62(52.1)$ & & & \\
\hline
\end{tabular}

ANRI, aspartate aminotransferase/neutrophil count ratio index; ICC, intrahepatic cholangiocarcinoma; HBsAg, hepatitis B surface antigen; $\mathrm{W}+\mathrm{M}$, well+moderated differentiation; $\mathrm{P}$, poor differentiation; $\mathrm{WBC}$, white blood cell; AST, aspartate aminotransferase; ALT, alanine transaminase; $\gamma$-GT, $\gamma$-glutamyl transpeptidase; AFP, $\alpha$-fetoprotein; CEA, carcinoembryonic antigen; CA19-9, carbohydrate antigen 19-9. ${ }^{a}$ Medians were used for cut-off values, b) According to the World Health Organization

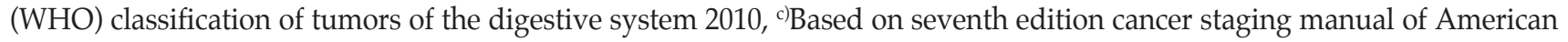
Joint Committee on Cancer.

\section{Results}

\section{Demographic and clinicopathological characteristics}

This study included 99 males $(53.8 \%)$ and 85 females $(46.2 \%)$. The mean age of the cohort was 56.0 years (range, 24 to 82 years). There were 163 patients $(88.6 \%)$ who developed tumor recurrence and 152 patients $(82.6 \%)$ died during the observation period. The mean tumor size was $6.0 \mathrm{~cm}$ (range,
1.0 to $20.0 \mathrm{~cm})$ at the greatest diameter. Elevated serum CA19$9(\geq 35.0 \mathrm{U} / \mathrm{mL})$ was observed in 119 patients $(64.7 \%)$, and 63 patients $(34.2 \%)$ had multiple tumor masses. Serum hepatitis B surface antigen ( $\mathrm{HBsAg}$ ) was positive in 51 patients $(27.7 \%)$, and cirrhosis existed in 46 patients (25.0\%). Additionally, 28 patients had a past history of surgery due to hepatolithiasis. Tumor differentiation was defined according to the World Health Organization classification of tumors of the digestive system 2010 [10]. Tumors from 125 patients $(67.9 \%)$ were well moderated, and those from 59 patients $(32.1 \%)$ were poorly 
A
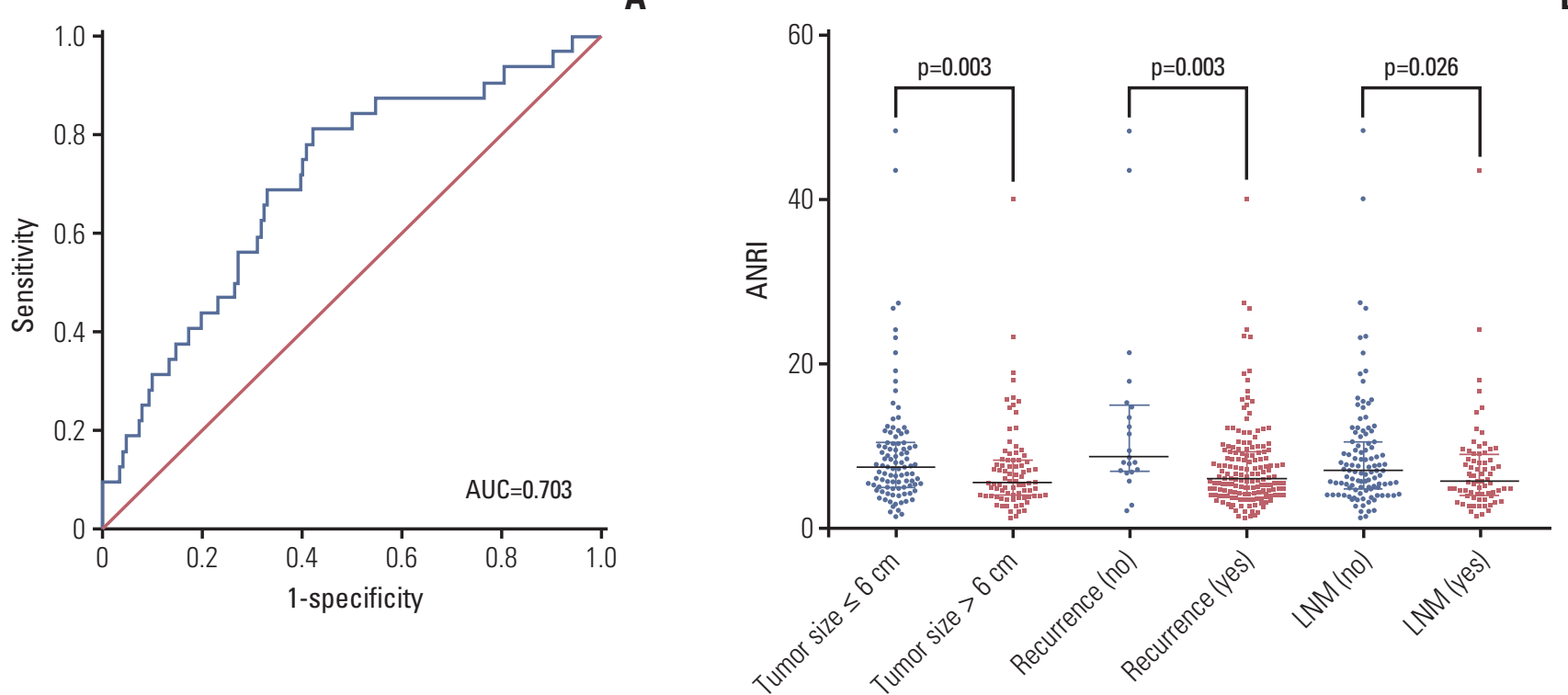

Fig. 1. Receiver operating characteristic (ROC) curve and distribution of preoperative aspartate aminotransferase/neutrophil count ratio index (ANRI) in different intrahepatic cholangiocarcinoma (ICC) subgroups. (A) ROC analysis was performed to determine the optimal cut-off value of ANRI in patients with ICC after hepatectomy (cut-off value $=6.7$ ). The area under the ROC curve for survival status was 0.703 (95\% confidence interval, 0.604 to $0.802 ; \mathrm{p}<0.001$ ), with a sensitivity of $81.3 \%$ and a specificity of $57.9 \%$. (B) Comparison of preoperative ANRI in ICC subgroups, stratified by tumor size, recurrence, and lymph node metastasis (LNM). The proportions of ICC patients with declined preoperative ANRI along with tumor size $>6 \mathrm{~cm}$, recurrence, and with LNM are much higher than those with tumor size $\leq 6 \mathrm{~cm}$, without recurrence, and without LNM, respectively (all $p<0.05$, Mann-Whitney $\mathrm{U}$ test). AUC, area under the curve.

differentiated. In addition, 79 patients $(42.9 \%)$ were in TNM stage I+II and 105 patients $(57.1 \%)$ were in TNM stage III+IV (Table 1).

\section{Determination of an ANRI cut-off value}

ANRI was calculated using the formula: ANRI=(AST value/neutrophil count) $\times 10^{9} / \mathrm{U}$. According to ROC curve analysis, the optimal cut-off value of ANRI was 6.7 for predicting postoperative prognosis. The area under ROC curve for survival status was 0.703 , with a $95 \%$ CI of $0.604-0.802$ $(\mathrm{p}<0.001)$ (Fig. 1A). The maximal Youden Index (sensitivity+ specifcity-1) showed a sensitivity of $81.3 \%$ and a specificity of $57.9 \%$. Subsequently, all patients were divided into two groups: a high ANRI group $(>6.7, \mathrm{n}=90)$ and low ANRI group $(\leq 6.7, n=94)$.

\section{Relationship between ANRI and clinicopathological char- acteristics in ICC}

The relationships between preoperative ANRI and clinicopathological variables were summarized in Table 1 . The results showed that preoperative ANRI was related to tumor size $\left(\chi^{2}=4.980, p=0.026\right)$, tumor recurrence $\left(\chi^{2}=12.848, p<0.001\right)$, white blood cell $\left(\chi^{2}=21.971, \mathrm{p}<0.001\right)$, neutrophil count $\left(\chi^{2}=26.679, \mathrm{p}<0.001\right), \operatorname{AST}\left(\chi^{2}=48.122, \mathrm{p}<0.001\right)$, and alanine transaminase $\left(\chi^{2}=18.952, p<0.001\right)$. However, there were no significance associations of ANRI with age, sex, cirrhosis, HBsAg, tumor number, tumor differentiation, lymph node metastasis, vascular invasion, TNM stage, and CA19-9 (all $\mathrm{p}>0.05$ ) (Table 1 ).

\section{Distribution of ANRI according to tumor size, recurrence, and lymph node metastasis}

The results indicated that preoperative ANRI in ICC patients with a tumor size $>6 \mathrm{~cm}$ was markedly lower than those with a tumor size $\leq 6 \mathrm{~cm}$ ( $\mathrm{p}=0.003$ ) (Fig. 1B). This tendency also existed in ICC patients with tumor recurrence and lymph node metastasis, compared to those without tumor recurrence and lymph node metastasis $(\mathrm{p}=0.003$ and $\mathrm{p}=0.026$, respectively) (Fig. 1B). 
Table 2. Univariate analyses for prognostic factors of patients with ICC $(n=184)$

\begin{tabular}{|c|c|c|c|c|c|c|c|}
\hline \multirow{2}{*}{ Category } & \multirow{2}{*}{ No. } & \multicolumn{2}{|c|}{ OS } & \multirow{2}{*}{ p-value } & \multicolumn{2}{|c|}{ DFS } & \multirow{2}{*}{ p-value } \\
\hline & & $1-Y e a r(\%)$ & 3-Year $(\%)$ & & $1-Y e a r(\%)$ & 3-Year $(\%)$ & \\
\hline \multicolumn{8}{|l|}{ Age (yr) } \\
\hline$\leq 60$ & 117 & 34.2 & 13.4 & 0.723 & 20.5 & 10.9 & 0.437 \\
\hline$>60$ & 67 & 41.8 & 11.2 & & 25.4 & 11.9 & \\
\hline \multicolumn{8}{|l|}{ Sex } \\
\hline Female & 85 & 47.1 & 15.6 & 0.014 & 30.6 & 14.6 & 0.035 \\
\hline Male & 99 & 28.3 & 8.1 & & 15.2 & 8.5 & \\
\hline \multicolumn{8}{|c|}{ Preoperative symptom } \\
\hline No & 51 & 41.2 & 16.7 & 0.464 & 27.5 & 10.5 & 0.575 \\
\hline Yes & 133 & 35.3 & 10.1 & & 20.3 & 11.4 & \\
\hline \multicolumn{8}{|l|}{ Cirrhosis } \\
\hline No & 138 & 39.1 & 12.6 & 0.303 & 23.9 & 11.7 & 0.268 \\
\hline Yes & 46 & 30.4 & 8.6 & & 17.4 & 9.9 & \\
\hline \multicolumn{8}{|l|}{ HBsAg } \\
\hline Negative & 133 & 36.8 & 13.1 & 0.916 & 23.3 & 11.9 & 0.465 \\
\hline Positive & 51 & 37.3 & 9.5 & & 19.6 & 9.8 & \\
\hline \multicolumn{8}{|l|}{ Child-Pugh class } \\
\hline A & 164 & 36.0 & 11.3 & 0.272 & 20.7 & 9.5 & 0.148 \\
\hline $\mathrm{B}$ & 20 & 45.0 & 25.0 & & 35.0 & 16.7 & \\
\hline \multicolumn{8}{|l|}{ No. of tumors } \\
\hline Single & 121 & 43.0 & 15.3 & 0.003 & 28.1 & 13.8 & 0.001 \\
\hline Multiple & 63 & 25.4 & 5.5 & & 11.1 & 6.3 & \\
\hline \multicolumn{8}{|l|}{ Tumor size (cm) } \\
\hline$\leq 6$ & 97 & 46.4 & 17.6 & 0.006 & 33.0 & 16.2 & $<0.001$ \\
\hline$>6$ & 87 & 26.4 & 7.9 & & 10.3 & 5.7 & \\
\hline \multicolumn{8}{|c|}{ Tumor differentiation } \\
\hline Well+moderated & 125 & 42.4 & 16.4 & 0.002 & 25.6 & 13.20 & 0.02 \\
\hline Poor & 59 & 25.4 & 6.1 & & 15.3 & 7.60 & \\
\hline \multicolumn{8}{|l|}{ Capsulation } \\
\hline Noncapsulated & 111 & 35.1 & 8.4 & 0.206 & 21.6 & 9.30 & 0.647 \\
\hline Capsulated & 73 & 39.7 & 17.7 & & 23.3 & 14.10 & \\
\hline \multicolumn{8}{|l|}{ Vascular invasion } \\
\hline No & 173 & 38.2 & 13.6 & 0.112 & 22.5 & 12.10 & 0.114 \\
\hline Yes & 11 & 18.2 & 0.0 & & 18.2 & 0.0 & \\
\hline \multicolumn{8}{|l|}{ TNM } \\
\hline I+II & 79 & 46.8 & 18.2 & 0.002 & 32.9 & 19.6 & 0.003 \\
\hline III+IV & 105 & 29.5 & 6.1 & & 14.3 & 5.3 & \\
\hline \multicolumn{8}{|l|}{ Resection margin } \\
\hline R0 & 108 & 42.6 & 20.1 & 0.001 & 28.7 & 17.0 & 0.001 \\
\hline R1 & 76 & 28.9 & 0.0 & & 13.2 & 2.60 & \\
\hline \multicolumn{8}{|c|}{ Lymph node metastasis } \\
\hline No & 111 & 43.2 & 17.7 & 0.003 & 29.7 & 15.5 & 0.011 \\
\hline Yes & 73 & 27.4 & 3.9 & & 11.0 & 5.1 & \\
\hline Biliary-intestinal an & & & & & & & \\
\hline No & 158 & 38.0 & 13.0 & 0.504 & 23.4 & 11.2 & 0.607 \\
\hline Yes & 26 & 30.8 & 13.5 & & 15.4 & 11.5 & \\
\hline Complication & & & & & & & \\
\hline No & 155 & 38.1 & 12.8 & 0.389 & 23.9 & 11.8 & 0.127 \\
\hline Yes & 29 & 31.0 & 23.0 & & 13.8 & 9.2 & \\
\hline
\end{tabular}

(Continued to the next page) 
Table 2. Continued

\begin{tabular}{|c|c|c|c|c|c|c|c|}
\hline \multirow{2}{*}{ Category } & \multirow{2}{*}{ No. } & \multicolumn{2}{|c|}{ OS } & \multirow{2}{*}{ p-value } & \multicolumn{2}{|c|}{ DFS } & \multirow{2}{*}{ p-value } \\
\hline & & $1-Y e a r(\%)$ & 3-Year $(\%)$ & & $1-Y e a r(\%)$ & 3-Year $(\%)$ & \\
\hline \multicolumn{8}{|c|}{ Intraoperative blood loss $(\mathrm{mL})^{\mathrm{a})}$} \\
\hline$\leq 400$ & 104 & 42.3 & 13.5 & 0.068 & 25.0 & 12.4 & 0.024 \\
\hline$>400$ & 80 & 30.0 & 12.1 & & 18.7 & 10.0 & \\
\hline \multicolumn{8}{|c|}{ WBC $\left(\times 10^{9} / \mathrm{L}\right)$} \\
\hline$\leq 10$ & 148 & 39.2 & 13.9 & 0.214 & 24.3 & 11.9 & 0.088 \\
\hline$>10$ & 36 & 27.8 & 10.0 & & 13.9 & 8.3 & \\
\hline \multicolumn{8}{|c|}{ Neutrophil $\left(\times 10^{9} / \mathrm{L}\right)^{\mathrm{a})}$} \\
\hline$\leq 4.55$ & 93 & 44.1 & 17.1 & 0.034 & 28.0 & 13.7 & 0.031 \\
\hline$>4.55$ & 91 & 29.7 & 9.1 & & 16.5 & 8.8 & \\
\hline \multicolumn{8}{|c|}{ Platelet $\left(\times 10^{9} / \mathrm{L}\right)$} \\
\hline$\leq 300$ & 139 & 37.4 & 13.0 & 0.754 & 22.3 & 11.0 & 0.963 \\
\hline$>300$ & 45 & 35.6 & 14.7 & & 22.2 & 12.3 & \\
\hline \multicolumn{8}{|l|}{ AST (U/L) } \\
\hline$\leq 37$ & 133 & 36.1 & 8.9 & 0.284 & 21.1 & 7.6 & 0.425 \\
\hline$>37$ & 51 & 39.2 & 20.3 & & 25.5 & 16.3 & \\
\hline \multicolumn{8}{|l|}{ ALT (U/L) } \\
\hline$\leq 80$ & 158 & 36.7 & 9.8 & 0.083 & 20.3 & 8.5 & 0.092 \\
\hline$>80$ & 26 & 38.5 & 34.6 & & 34.6 & 26.9 & \\
\hline \multicolumn{8}{|c|}{$\gamma$-GT (U/L) } \\
\hline$\leq 50$ & 50 & 48.0 & 12.3 & 0.275 & 28.0 & 9.9 & 0.292 \\
\hline$>50$ & 134 & 32.8 & 13.5 & & 20.1 & 10.1 & \\
\hline \multicolumn{8}{|l|}{$\operatorname{AFP}(\mu \mathrm{g} / \mathrm{L})$} \\
\hline$\leq 200$ & 176 & 36.9 & 13.1 & 0.658 & 22.7 & 11.2 & 0.259 \\
\hline$>200$ & 8 & 37.5 & 0.0 & & 12.5 & 0.0 & \\
\hline \multicolumn{8}{|c|}{ CEA $(\mu \mathrm{g} / \mathrm{L})$} \\
\hline$\leq 5$ & 107 & 44.9 & 17.7 & 0.008 & 29.9 & 13.4 & 0.001 \\
\hline$>5$ & 77 & 26.0 & 6.6 & & 11.7 & 5.5 & \\
\hline \multicolumn{8}{|c|}{ CA19-9 (U/mL) } \\
\hline$\leq 35$ & 65 & 43.1 & 12.0 & 0.324 & 24.6 & 8.3 & 0.554 \\
\hline$>35$ & 119 & 33.6 & 13.9 & & 21.0 & 11.2 & \\
\hline \multicolumn{8}{|l|}{ ANRI } \\
\hline$\leq 6.7$ & 94 & 30.9 & 3.8 & 0.004 & 13.8 & 3.2 & 0.003 \\
\hline$>6.7$ & 90 & 43.3 & 26.6 & & 31.1 & 20.9 & \\
\hline
\end{tabular}

ICC, intrahepatic cholangiocarcinoma; OS, overall survival; DFS, disease-free survival; $\mathrm{HBsAg}$, hepatitis B surface antigen; WBC, white blood cell; AST, aspartate aminotransferase; ALT, alanine transaminase; $\gamma$-GT, $\gamma$-glutamyl transpeptidase; AFP, $\alpha$-fetoprotein; CEA, carcinoembryonic antigen; CA19-9, carbohydrate antigen 19-9; ANRI, aspartate aminotransferase/neutrophil count ratio index. ${ }^{a}$ Medians were used for cut-off values.

\section{Prognostic factors for ICC}

In this study, the 1-year and 3-year OS rates of all patients were $37.0 \%$ and $13.0 \%$, respectively. The 1-year and 3-year DFS rates were $22.3 \%$ and $11.3 \%$, respectively. The median OS and DFS times were 10.0 and 4.0 months, respectively. Univariate analysis and the multivariate Cox's proportional hazard regression model analysis were performed to identify the risk factors of prognosis. Univariate analysis revealed that preoperative ANRI $(p=0.004)$, sex $(p=0.014)$, tumor number $(\mathrm{p}=0.003)$, tumor size $(\mathrm{p}=0.006)$, tumor differentiation $(\mathrm{p}=0.002)$, TNM stage $(\mathrm{p}=0.002)$, resection margin $(\mathrm{p}=0.001)$, lymph node metastasis $(\mathrm{p}=0.003)$, neutrophil $(\mathrm{p}=0.034)$, and CEA ( $p=0.008)$ were prognostic factors for OS among the ICC cohort (Table 2). The preoperative ANRI $(p=0.003)$, sex $(\mathrm{p}=0.035)$, tumor number $(\mathrm{p}=0.001)$, tumor size $(\mathrm{p}<0.001)$, 
Table 3. Multivariate analyses for independent prognostic factors of patients with ICC $(\mathrm{n}=184)$

\begin{tabular}{|c|c|c|c|c|}
\hline \multirow{2}{*}{ Category } & \multicolumn{2}{|c|}{ OS } & \multicolumn{2}{|c|}{ DFS } \\
\hline & HR ( $95 \%$ CI) & p-value & HR $(95 \%$ CI) & p-value \\
\hline Tumor number (single vs. multiple) & $1.473(1.048-2.069)$ & 0.026 & - & - \\
\hline Tumor size $(\leq 6 \mathrm{~cm}$ vs. $>6 \mathrm{~cm})$ & $1.408(1.013-1.959)$ & 0.042 & $1.725(1.251-2.380)$ & 0.001 \\
\hline Tumor differentiation (W+M vs. $\mathrm{P}$ ) & $1.687(1.202-2.366)$ & 0.002 & $1.501(1.078-2.090)$ & 0.016 \\
\hline Resection margin (R0 vs. R1) & $0.556(0.401-0.770)$ & $<0.001$ & $0.580(0.421-0.800)$ & 0.001 \\
\hline ANRI (> 6.7 vs. $\leq 6.7)$ & $0.671(0.482-0.934)$ & 0.018 & $0.664(0.483-0.911)$ & 0.011 \\
\hline
\end{tabular}

Variables with $\mathrm{p}<0.05$ calculated by Kaplan-Meier method (log-rank test) were used in multivariate analysis (Cox proportional hazards model, Backward stepwise). ICC, intrahepatic cholangiocarcinoma; OS, overall survival; DFS, disease-free survival; $\mathrm{HR}$, hazard ratio; $\mathrm{CI}$, confidence interval; $\mathrm{W}+\mathrm{M}$, well+moderated differentiation; $\mathrm{P}$, poor differentiation; ANRI, aspartate aminotransferase/ neutrophil count ratio index.
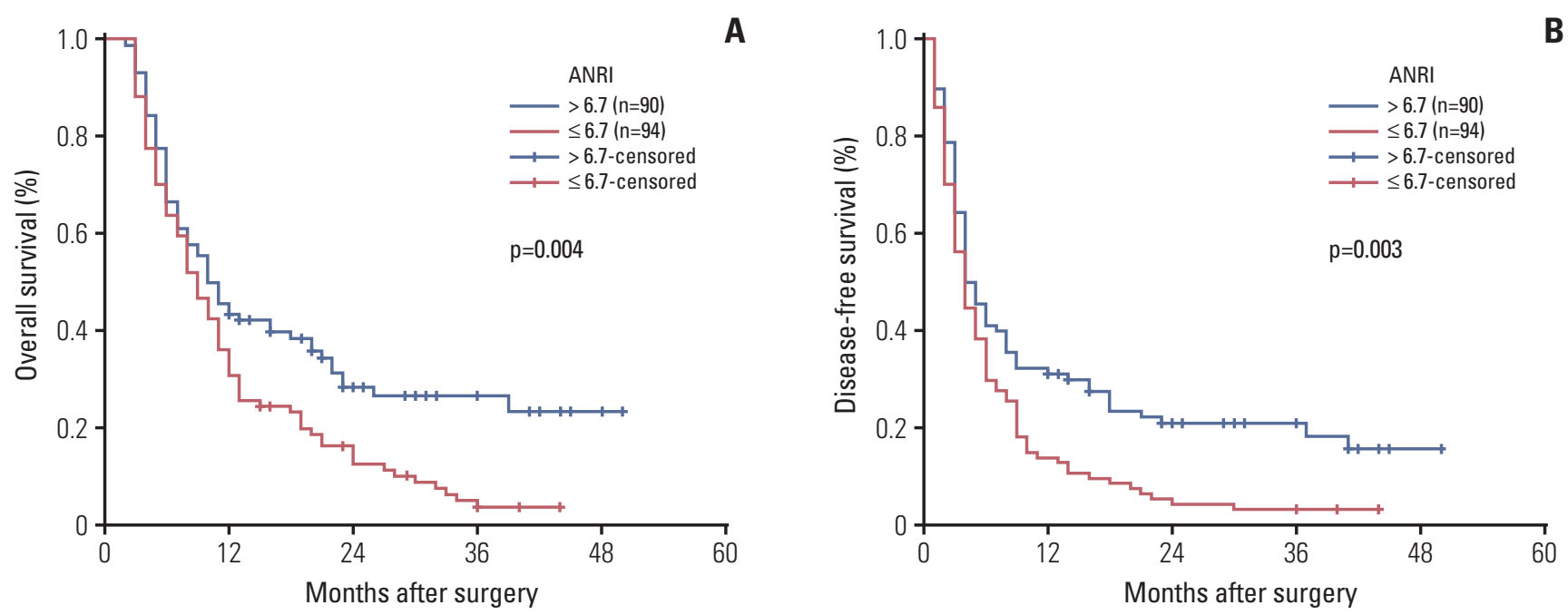

Fig. 2. Kaplan-Meier survival curves of overall survival (A) and disease-free survival (B) for intrahepatic cholangiocarcinoma patients with aspartate aminotransferase/neutrophil count ratio index (ANRI) > 6.7 and ANRI $\leq 6.7$ in the entire cohort. p-values were obtained by log-rank tests.

tumor differentiation $(\mathrm{p}=0.020)$, TNM stage $(\mathrm{p}=0.003)$, resection margin $(p=0.001)$, lymph node metastasis $(p=0.011)$, intraoperative blood loss $(\mathrm{p}=0.024)$, neutrophil $(\mathrm{p}=0.031)$, and CEA ( $p=0.001)$ were prognostic predictors for DFS in patients with ICC after hepatectomy (Table 2). After multivariate analysis, the results indicated that ANRI, tumor number, tumor size, tumor differentiation, and resection margin were significant independent prognostic markers of OS (all $\mathrm{p}<0.05$ ), while ANRI, tumor size, differentiation, and resection margin were independent predictors of DFS (all $p<0.05$ ) (Table 3).

\section{OS and DFS according to ANRI}

Kaplan-Meier method was performed to clarify the influence of preoperative ANRI on patient survival. The results indicated that the 1-year and 3-year OS rates of the ANRI $\leq 6.7$ group were markedly lower than those of the ANRI $>6.7$ group $(30.9 \%$ and $3.8 \%$ vs. $43.3 \%$ and $26.6 \%$, respectively, $\mathrm{p}=0.004$ ) (Table 2, Fig. 2A), while the 1-year and 3-year DFS rates of the ANRI $\leq 6.7$ group were also significantly lower than those of the ANRI > 6.7 group $(13.8 \%$ and $3.2 \%$ vs. $31.1 \%$ and $20.9 \%$, respectively, $\mathrm{p}=0.003$ ) (Table 2 , Fig. 2B). Therefore, a low preoperative ANRI level was correlated with a poor survival outcome in patients with ICC after hepatectomy. 


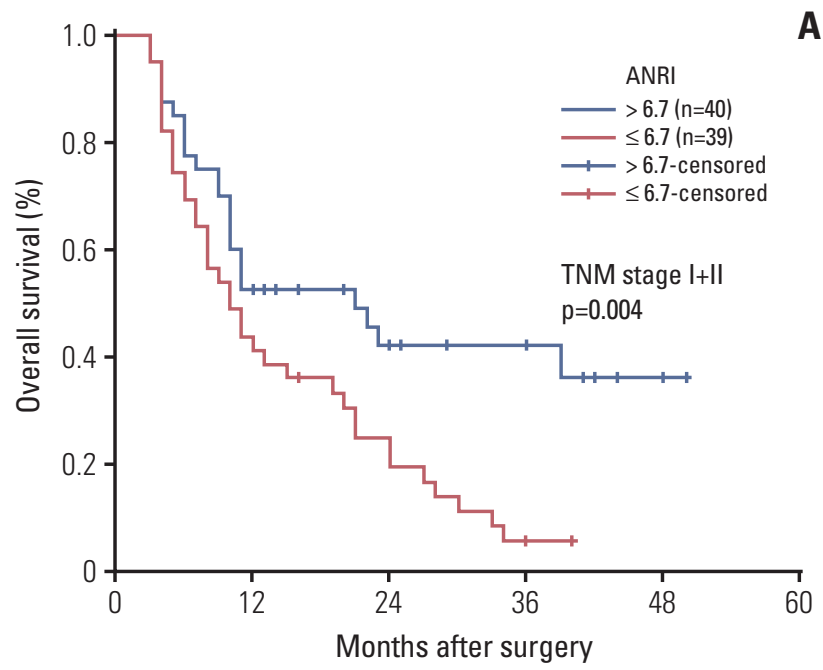

A
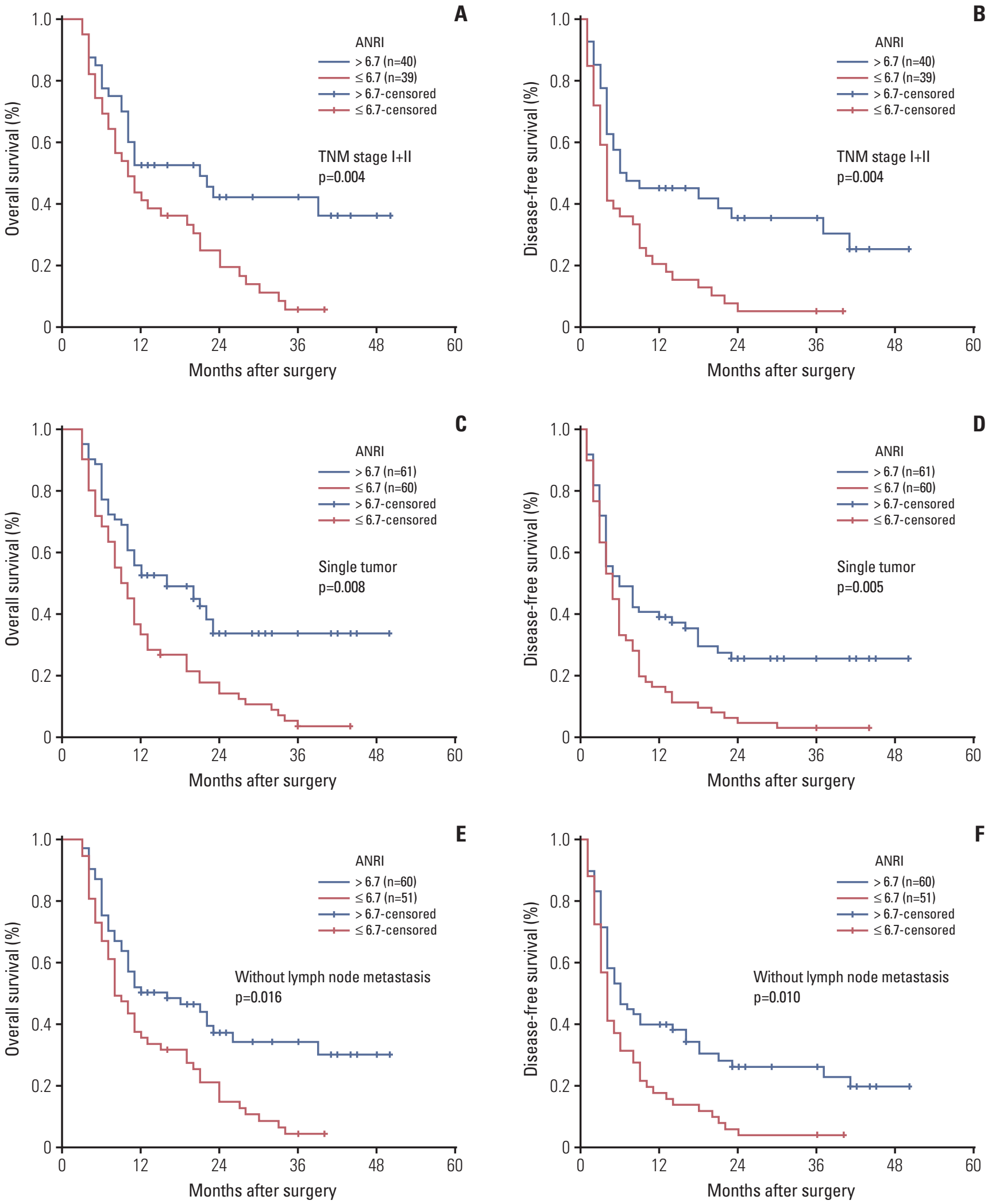

Fig. 3. Kaplan-Meier survival curves of patients with intrahepatic cholangiocarcinoma after hepatectomy stratified by TNM stage I+II (A, B), single tumor status (C, D), and without lymph node metastasis (E, F). p-values were obtained by log-rank tests. ANRI, aspartate aminotransferase/neutrophil count ratio index. 


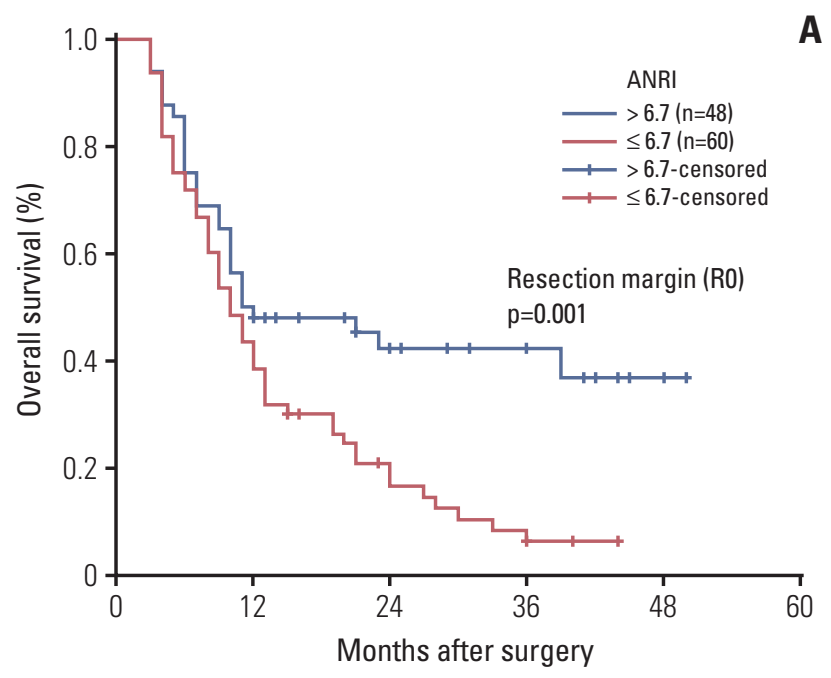

A
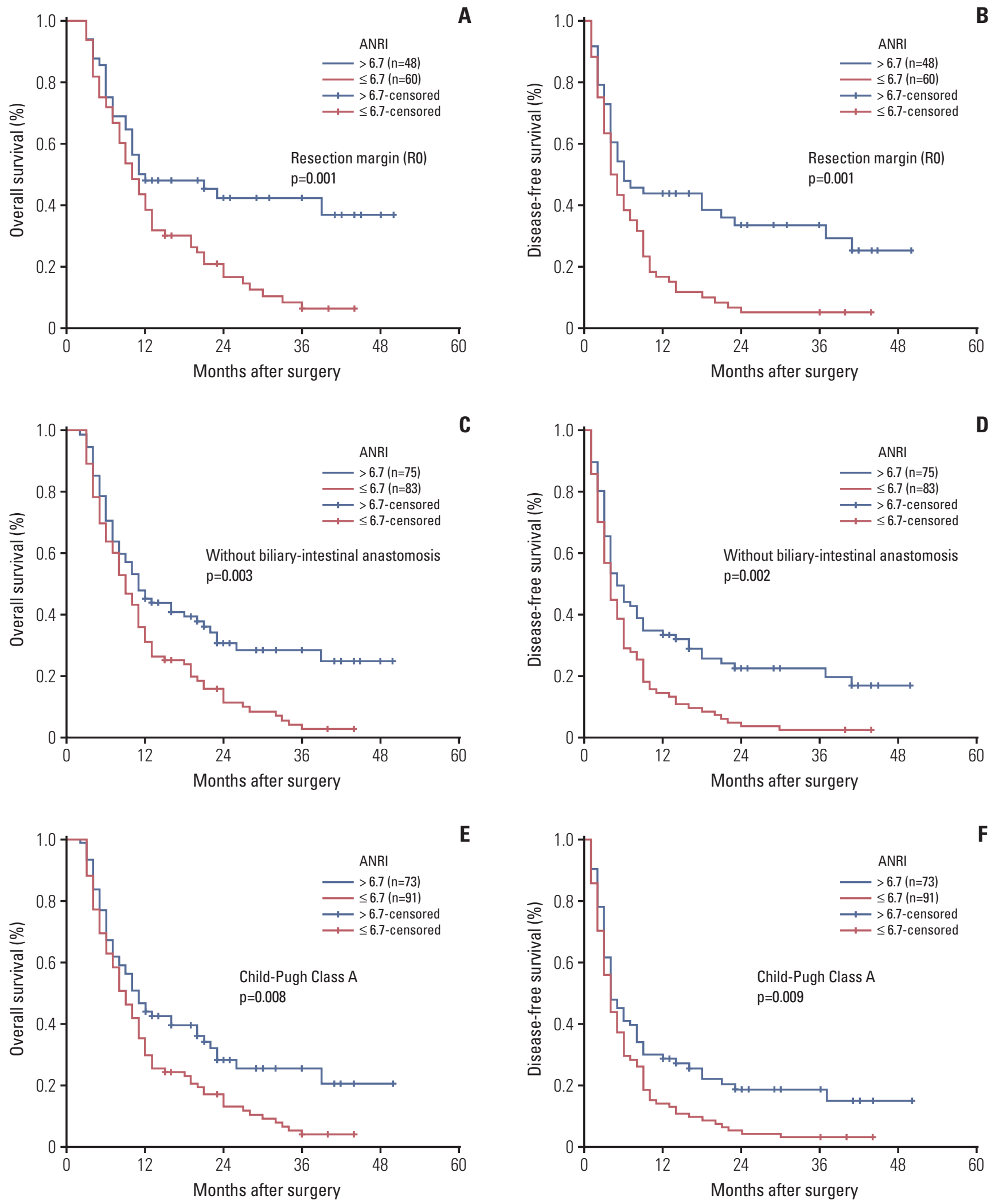

E

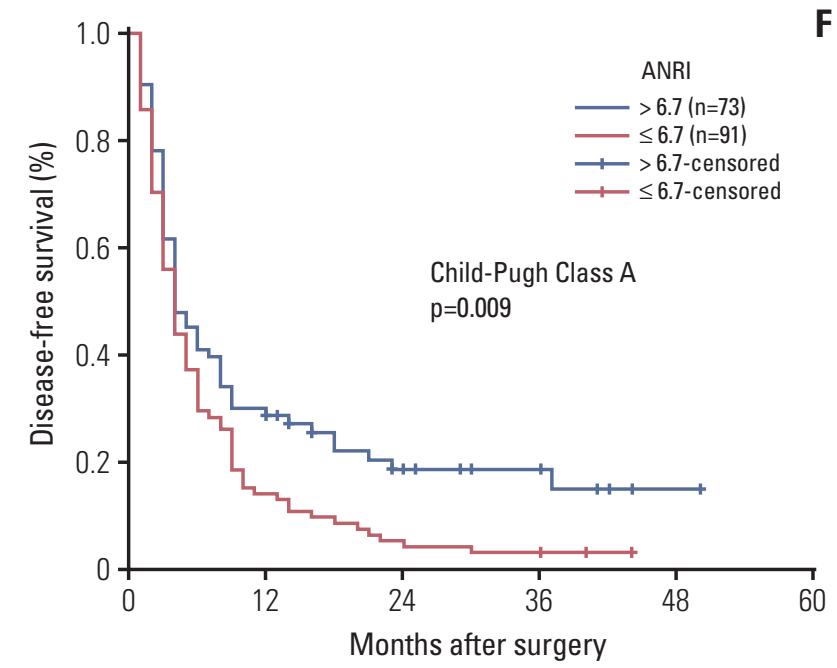

Fig. 4. Kaplan-Meier survival curves of patients with intrahepatic cholangiocarcinoma after hepatectomy stratified by R0 resection margin $(\mathrm{A}, \mathrm{B})$, without biliary-intestinal anastomosis $(\mathrm{C}, \mathrm{D})$, and Child-Pugh Class A (E, F). p-values were obtained by log-rank tests. ANRI, aspartate aminotransferase/ neutrophil count ratio index. 


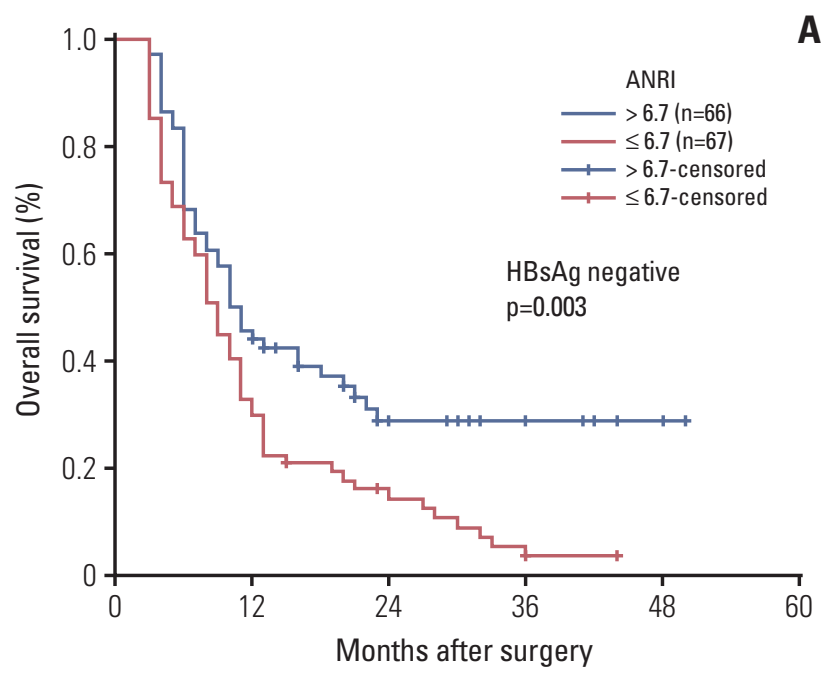

A
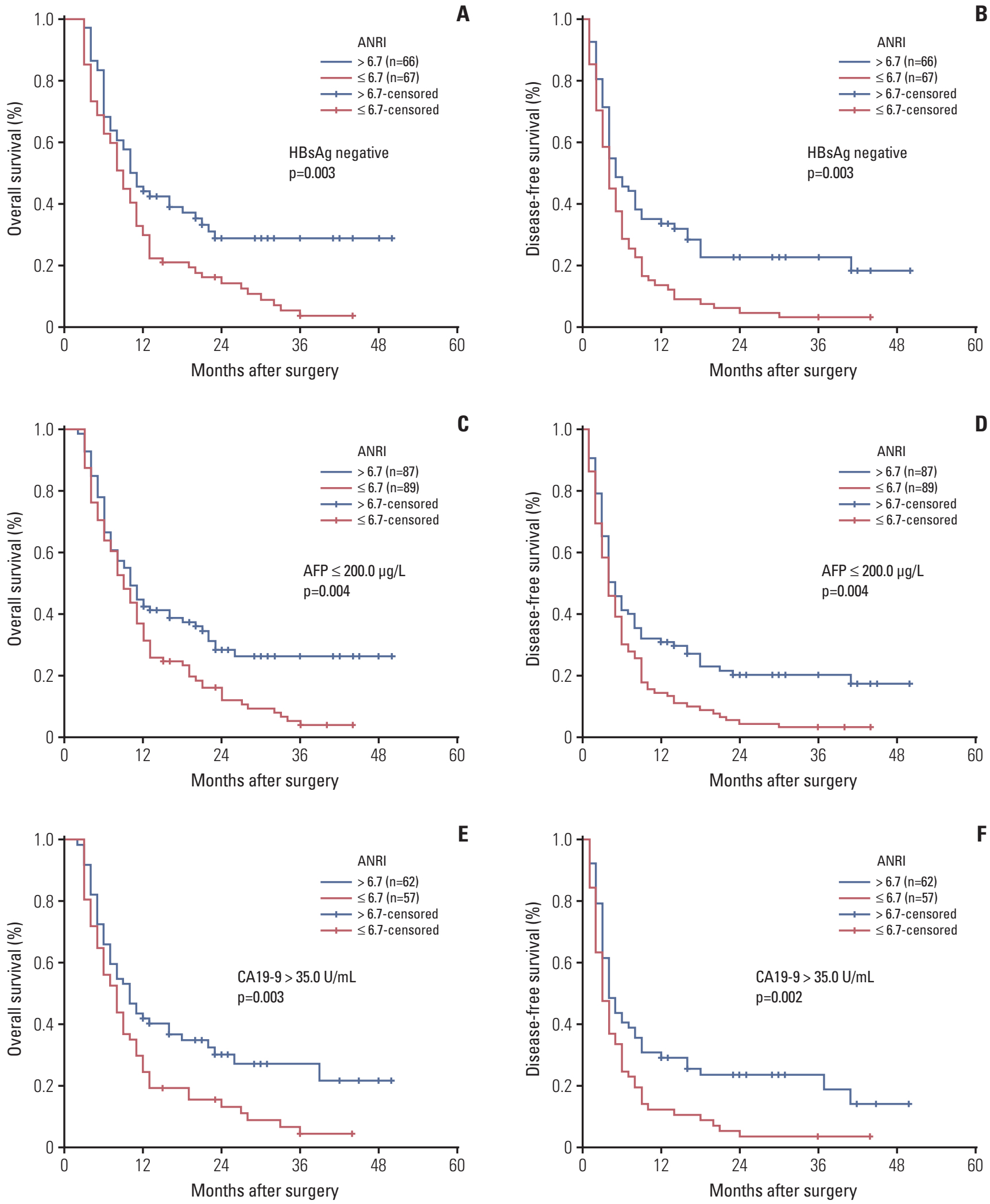

$\mathbf{E}$

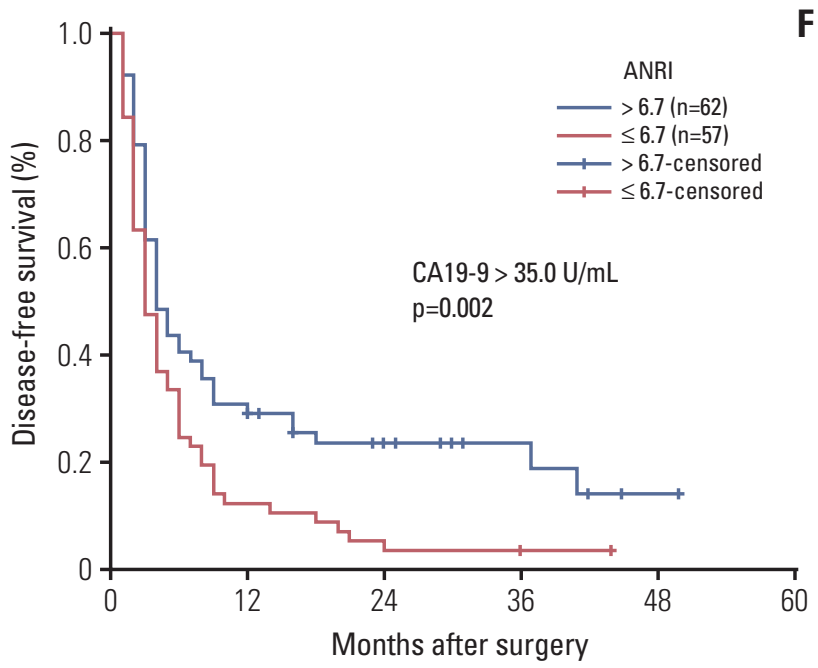

Fig. 5. Kaplan-Meier survival curves of patients with intrahepatic cholangiocarcinoma after hepatectomy stratified by serum hepatitis B surface antigen (HBsAg) negative (A, B), $\alpha$-fetoprotein (AFP) $\leq 200.0 \mu \mathrm{g} / \mathrm{L}$ (C, D), and carbohydrate antigen 19-9 (CA19-9) $>35.0 \mathrm{U} / \mathrm{mL}$ (E, F). p-values were obtained by log-rank tests. ANRI, aspartate aminotransferase/neutrophil count ratio index. 


\section{Prognostic values of ANRI in various subgroups of ICC}

The research above confirmed that preoperative ANRI $\leq 6.7$ was significantly correlated with lower OS and DFS rates in patients with ICC. We further evaluated the prognostic significance of preoperative ANRI in various subgroups of ICC patients. The data showed that preoperative ANRI was a prognostic predictor for OS $(p=0.004)$ and DFS $(p=0.004)$ in ICC patients with TNM stage of I+II (Fig. 3A and B). This predictive value of declined preoperative ANRI for poorer OS and DFS also existed in the subgroups of single tumor, without lymph node metastasis, $\mathrm{R} 0$ resection margin, without biliary-intestinal anastomosis, Child-Pugh Class A, serum HBsAg negative, AFP $\leq 200.0 \mu \mathrm{g} / \mathrm{L}$, and CA19-9 > 35.0 U/mL (all $\mathrm{p}<0.05)$ (Figs. 3C-F, 4, 5). These results suggested that preoperative ANRI was a sensitive parameter to predict the survival of ICC patients after hepatic resection, especially in various subgroups of patients with ICC whose prognosis is difficult to estimate.

\section{Discussion}

Tumor recurrence or metastasis severely influenced the prognosis of ICC after hepatectomy, a simple and effective method to identify surgical patients at high risk of recurrence is of great value for clinical work. In this study, we find ANRI to be a reliable non-invasive prognostic biomarker for ICC patients.

Inflammation and inflammatory biomarkers play a central role in tumor progression, associating with tumor proliferation, survival and migration [11]. Neutrophils are a critical component of the systemic inflammatory response and several studies have verified that the neutrophil count or neutrophil-lymphocyte ratio index were predictors of prognosis in various malignancies $[7,12,13]$. In the present study, we found that preoperative declined ANRI $(\leq 6.7)$ showed earlier recurrence and poorer OS in ICC patients after hepatic resection. Additionally, our data suggested that ANRI was negatively related to tumor size and recurrence. These results indicated that ANRI could reflect not only the hepatocyte injury but also the tumor burden and disease development. Both univariate analysis and multivariate analysis showed that tumor size $>6 \mathrm{~cm}$, poor differentiation, $\mathrm{R} 1$ resection margin and declined ANRI were independent risk factors for both poor OS and DFS of ICC patients after hepatectomy.

The results of further analysis showed that preoperative ANRI had a significant predictive value for both OS and DFS in ICC patients with TNM stage I+II, meaning that ANRI could predict recurrence of early ICC. As one of the risk fac- tors of ICC, chronic hepatitis B virus infection impacted prognosis of ICC following resection [14]. It is usually more difficult to estimate the survival of serum HBsAg-negative patients. However, the data in this study indicated that ANRI shows its markedly prognostic value in those ICC patients. Furthermore, in subgroups with a single tumor, without lymph node metastasis, a R0 resection margin, without biliary-intestinal anastomosis, Child-Pugh Class A, an $\mathrm{AFP} \leq 200.0 \mu \mathrm{g} / \mathrm{L}$ and an elevated CA19-9 level, preoperative ANRI $\leq 6.7$ also shows its significance in predicting poorer OS and DFS.

These results showed that preoperative ANRI is a potential efficient prognostic indicator of survival in patients with ICC after hepatectomy. However, the exact reason for the association between declined preoperative ANRI and poor prognosis is not well understood. Our study showed that preoperative neutrophil count was markedly negatively correlated with ANRI, which means that patients with a lower ANRI have relative neutrophilia. The critical role of neutrophil in systemic inflammation and tumor progression might explain part of this phenomenon. Neutrophils were reported to facilitate tumor cell invasion and migration by secreting immune-reactive substances such as hepatocyte growth factor [15] and neutrophil elastase [16]. In addition, neutrophils can affect the prognosis of malignancy by directly down-regulating the cellular immunity function of the host [17]. ICC cells may recruit more neutrophils to the tumor foci by expressing higher levels of chemokine ligand 5 , which amplifies the inflammatory response and promotes ICC metastasis and recurrence [18]. Furthermore, increased neutrophils are regarded as an important composition of compartments for circulating vascular endothelial growth factor (VEGF) [19]. Patients with lower ANRI have relative neutrophilia and may have a higher level of circulating VEGF. It has been reported that autocrine VEGF/vascular endothelial growth factor receptor 2 signaling promotes growth of ICC cells, and apatinib inhibits anti-apoptotic cell proliferation by suppressing the autocrine VEGF signaling [20]. Therefore, apatinib may be considered as a candidate in the treatment of ICC patients with lower preoperative ANRI.

There are some limitations in the present study. First, it is a retrospective study of a single center and the sample size is relatively small. Second, our data is mainly based on ICC patients with predominant hepatitis $B$ virus infection and hepatolithiasis in China. Therefore, the results for application in Western populations with predominant primary sclerosing cholangitis [21] or hepatitis $C$ virus infection should be further studied.

In conclusion, our study showed that preoperative declined ANRI is a novel independent indicator for poor prognosis of ICC patients after hepatectomy. ICC patients with preoperative declined ANRI might benefit from close 
follow up and prophylactic personalized therapy.

\section{Conflicts of Interest}

Conflict of interest relevant to this article was not reported.

\section{Acknowledgments}

This study was supported by the Research Fund of the Young Teacher Culture Program (Grant number: 15ykpy15) and the Natural Science Foundation of Guangdong Province (Grant number: 2014A030313108).

\section{References}

1. Moeini A, Sia D, Bardeesy N, Mazzaferro V, Llovet JM. Molecular pathogenesis and targeted therapies for intrahepatic cholangiocarcinoma. Clin Cancer Res. 2016;22:291-300.

2. Bridgewater J, Galle PR, Khan SA, Llovet JM, Park JW, Patel $\mathrm{T}$, et al. Guidelines for the diagnosis and management of intrahepatic cholangiocarcinoma. J Hepatol. 2014;60:1268-89.

3. Jutric Z, Johnston WC, Hoen HM, Newell PH, Cassera MA, Hammill CW, et al. Impact of lymph node status in patients with intrahepatic cholangiocarcinoma treated by major hepatectomy: a review of the National Cancer Database. HPB (Oxford). 2016;18:79-87.

4. Spolverato G, Kim Y, Alexandrescu S, Marques HP, Lamelas J, Aldrighetti L, et al. Management and outcomes of patients with recurrent intrahepatic cholangiocarcinoma following previous curative-intent surgical resection. Ann Surg Oncol. 2016;23:235-43.

5. Poultsides GA, Zhu AX, Choti MA, Pawlik TM. Intrahepatic cholangiocarcinoma. Surg Clin North Am. 2010;90:817-37.

6. Kim HC, Nam CM, Jee SH, Han KH, Oh DK, Suh I. Normal serum aminotransferase concentration and risk of mortality from liver diseases: prospective cohort study. BMJ. 2004;328: 983.

7. Fu SJ, Shen SL, Li SQ, Hua YP, Hu WJ, Liang LJ, et al. Prognostic value of preoperative peripheral neutrophil-to-lymphocyte ratio in patients with $\mathrm{HBV}$-associated hepatocellular carcinoma after radical hepatectomy. Med Oncol. 2013;30:721.

8. Jin J, Zhu P, Liao Y, Li J, Liao W, He S. Elevated preoperative aspartate aminotransferase to lymphocyte ratio index as an independent prognostic factor for patients with hepatocellular carcinoma after hepatic resection. Oncotarget. 2015;6:1921727.

9. Ji F, Fu S, Guo Z, Pang H, Chen D, Wang X, et al. Prognostic significance of preoperative aspartate aminotransferase to neutrophil ratio index in patients with hepatocellular carcinoma after hepatic resection. Oncotarget. 2016;7:72276-89.

10. Bosman FT, Carneiro F, Hruban RH, Theise ND. WHO classification of tumours of the digestive system. 4th ed. Lyon: IARC Press; 2010.

11. Mantovani A, Allavena P, Sica A, Balkwill F. Cancer-related inflammation. Nature. 2008;454:436-44.
12. Nakashima H, Matsuoka Y, Yoshida R, Nagata M, Hirosue A, Kawahara K, et al. Pre-treatment neutrophil to lymphocyte ratio predicts the chemoradiotherapy outcome and survival in patients with oral squamous cell carcinoma: a retrospective study. BMC Cancer. 2016;16:41.

13. Park HS, Lee HS, Park JS, Park JS, Lee DK, Lee SJ, et al. Prognostic scoring index for patients with metastatic pancreatic adenocarcinoma. Cancer Res Treat. 2016;48:1253-63.

14. Zhang L, Cai JQ, Zhao JJ, Bi XY, Tan XG, Yan T, et al. Impact of hepatitis $B$ virus infection on outcome following resection for intrahepatic cholangiocarcinoma. J Surg Oncol. 2010;101: 233-8.

15. Wislez M, Rabbe N, Marchal J, Milleron B, Crestani B, Mayaud $\mathrm{C}$, et al. Hepatocyte growth factor production by neutrophils infiltrating bronchioloalveolar subtype pulmonary adenocarcinoma: role in tumor progression and death. Cancer Res. 2003;63:1405-12.

16. Houghton AM, Rzymkiewicz DM, Ji H, Gregory AD, Egea EE, Metz HE, et al. Neutrophil elastase-mediated degradation of IRS-1 accelerates lung tumor growth. Nat Med. 2010;16: 219-23.

17. Teramukai S, Kitano T, Kishida Y, Kawahara M, Kubota K, Komuta $\mathrm{K}$, et al. Pretreatment neutrophil count as an independent prognostic factor in advanced non-small-cell lung cancer: an analysis of Japan Multinational Trial Organisation LC00-03. Eur J Cancer. 2009;45:1950-8.

18. Zhou SL, Dai Z, Zhou ZJ, Chen Q, Wang Z, Xiao YS, et al. CXCL5 contributes to tumor metastasis and recurrence of intrahepatic cholangiocarcinoma by recruiting infiltrative intratumoral neutrophils. Carcinogenesis. 2014;35:597-605.

19. Kusumanto YH, Dam WA, Hospers GA, Meijer C, Mulder $\mathrm{NH}$. Platelets and granulocytes, in particular the neutrophils, form important compartments for circulating vascular endothelial growth factor. Angiogenesis. 2003;6:283-7.

20. Peng H, Zhang Q, Li J, Zhang N, Hua Y, Xu L, et al. Apatinib inhibits VEGF signaling and promotes apoptosis in intrahepatic cholangiocarcinoma. Oncotarget. 2016;7:17220-9.

21. Khan SA, Thomas HC, Davidson BR, Taylor-Robinson SD. Cholangiocarcinoma. Lancet. 2005;366:1303-14. 